\title{
In-situ TEM-STM Investigation of Conductance in Cu Atomic Wires
}

\author{
J.L. Rullan and K.A. Dunn
}

College of Nanoscale Science and Engineering, University at Albany - State University of New York, 251 Fuller Road, Albany, NY 12203

Nanotechnology derives both its promise and its difficulties from inherently nanoscale phenomena, which bring attendant challenges in characterization. Nanoscale metallic wires serve as a prime example, where simultaneous electrical and structural characterization pushes the boundaries of established techniques. For example, electrical measurements of nanowires drawn from a metallic surface using a scanning tunneling microscopy (STM) tip is possible, although structural information about the wire cannot be collected simultaneously [1-4]. Conversely, while the structure of nanowires formed by controlled electron-beam melting is accessible, it is cumbersome to attain electrical properties of the same nanowire [5]. However, the incorporation of an STM in a transmission electron microscopy (TEM) holder has enabled the electrical and atomic resolution structural analysis of a particular metal nanowire to proceed simultaneously [6]. Such a system has revealed, for example, quantized conductance in nanoscale gold wires [7-9], but very little work has done with other non-noble metal systems [10].

Employing a commercially available TEM-STM holder, we have applied this method to the investigation of the structural and electrical properties of $\mathrm{Cu}$ nanowires. Using conventional phosphoric acid electropolishing, $\mathrm{Cu}$ wires were thinned to a diameter of $\sim 100 \mathrm{~nm}$ (Fig. 1). Initial electrical contact using the TEM-STM holder resulted in a nonlinear response, suggesting the presence of an oxide or phosphide layer on the surface of the copper, although the EDS spectrum found no residual phosphorous contamination on the tip of the wire (Fig. 2). This barrier was overcome by applying a brief high bias pulse in conjunction with a forceful physical contact between tip and sample. After an Ohmic connection was made, the electron beam was used to irradiate the edges of the wire and further thin the wire. The result is shown in Fig. 3 with a wire necked down to about $\sim 10 \mathrm{~nm}$. The electrical characterization of this wire is shown in Fig. 4, which corresponds to a Sharvin resistance of $\sim 40 \Omega$, slightly higher than the expected value of $11 \Omega$, but consistent with reported values for gold nanowires ( $\sim 40$ for a $7.1 \mathrm{~nm}$ wire [8]). Crystallinity is preserved on either side of the necking region, but is difficult to determine whether the neck itself remains crystalline or the electron irradiation has distorted the structure of the material. The use of high resolution EELS to identify the possible migration of any incorporated impurities and influence of irradiation on the oxidation state of the metal will be discussed.

\section{References}

[1] J. I. Pascual, J. Mendez, J. Gomez-Herrero, A.M. Baro and N. Garcia, Phys. Rev. Lett. 71(12), 1852 (1993).

[2] L. Olesen, E. Laegsgaard, I. Stensgaard, F. Besenbacher, J. Schiotz, P. Toltze, K.W. Jacobsen and J. K. Norskov, Phys. Rev. Lett. 72(14), 2251 (1994).

[3] M. Brandbyge, J. Schiotz, M.R. Sorenssen, P. Stoltze, K. W. Jacobsen, J.K. Norskov, L. Olesen, E. Laegsgaard, I. Stensgaard, and F. Besenbacher, Phys. Rev. B 52(11), 8499 (1995).

[4] E. Scheer, N. Agrait, J.C. Cuevas, A. L. Yeyati, B. Ludoph, A. Marin-Rodero, G. R. Bollinger, J.M. van Ruitenbeek and C. Urbina, Nature 394, 154 (1998). 
[5] V. Rodrigues, T. Fuhrer, and D. Ugarte, Phy. Rev. Let. 85, 19 (2000)

[6] D. Erts, A. Lohmus, R. Lohmus, H. Olin Appl. Phys. A 72 [Suppl.], S71-S74 (2001)

[7] H. Ohnishi, Y. Kondo and K. Takayanagi, Nature 395, 780 (1998).

[8] D. Erts, H. Olin, L. Ryen, E. Olsson and A. Tholen, Phys. Rev. B 61(19), 12725 (2000).

[9] T. Kizuka, S. Deguchi, M. Naruse, S. Fujisawa, S. Sasaki, A. Yabe and Y. Enomoto, Proc. $15^{\text {th }}$ Internat. Congress of Electron Microscopy, 527 (2002).

[10] K.A. Dunn, J. Rullan and Y. Zhu, "In-situ TEM-STM Investigation of Conductance in Bimetallic Atomic Wires", Microscopy and Microanalysis 11 (Suppl. 2), 1508 (2005).

\section{Acknowledgements}

The authors gratefully acknowledge research funding through the SRC NY CAIST program under contract 1292.005. In addition, instrument support was provided in part through the DARPA and MARCO funded Interconnect Focus Center.

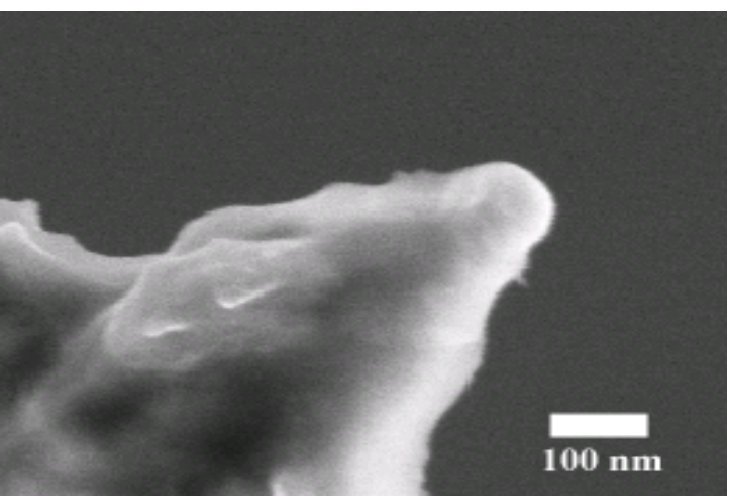

Fig. 1. Secondary electron image of $\mathrm{Cu}$ wire narrowed to $100 \mathrm{~nm}$ diameter using a phosphoric acid electropolish.

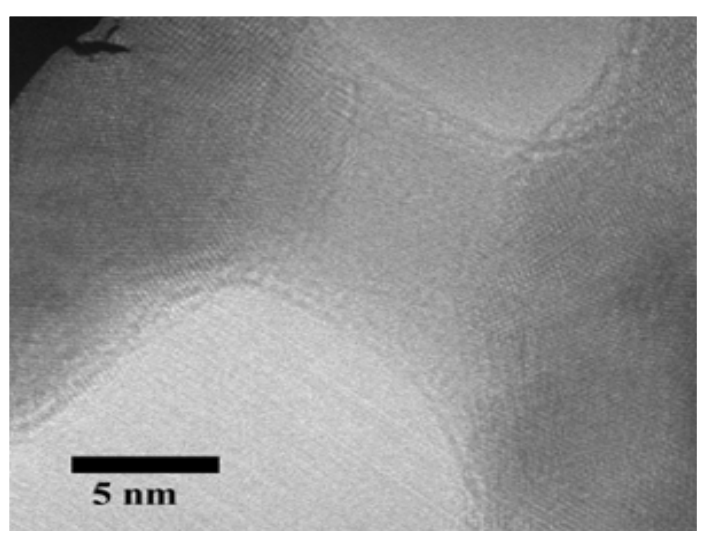

Fig. 3. TEM micrograph of a copper nanowire being electrically tested. Crystallinity preserved on either side of the neck, although the neck itself may have suffered damage from irradiation.

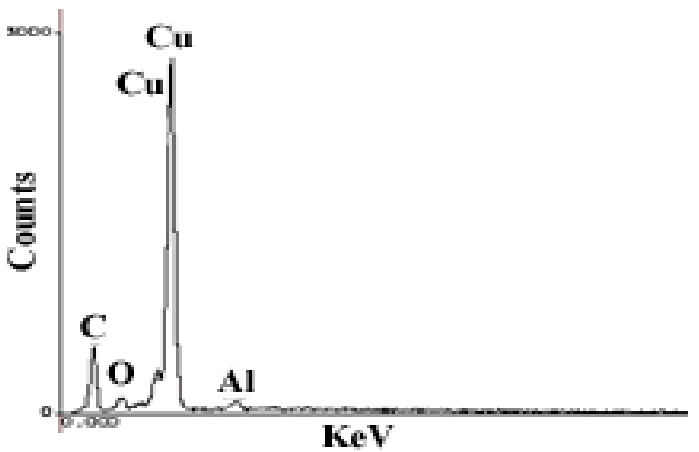

Fig. 2. EDS spectrum of a $\mathrm{Cu}$ wire postelectropolishing. No residual phosphorous contamination is evident.

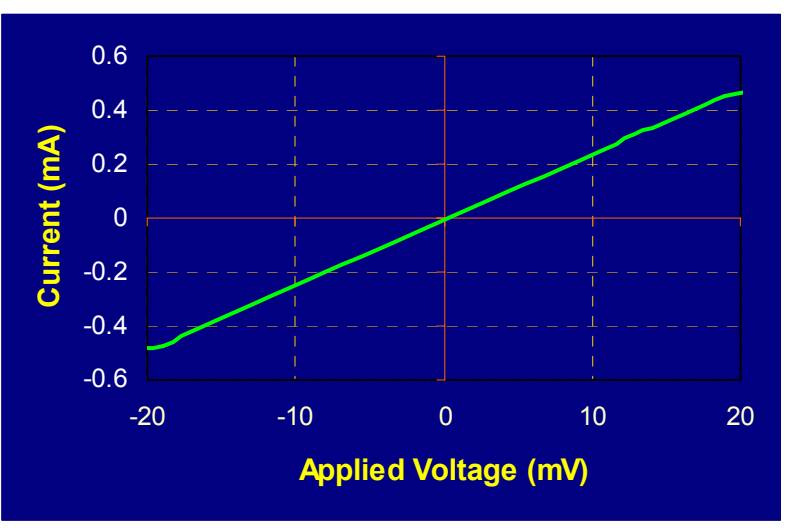

Fig. 4. Ohmic electrical response of a $\sim 10 \mathrm{~nm}$ diameter wire $(\mathrm{R} \sim 40 \Omega)$. While slightly higher than predicted $(\sim 11 \Omega)$, this value is consistent with previous observations in gold nanowires. 